\title{
Refractive Indices, Order Parameter and Optical Transmittance Studies of a Nematic Liquid Crystal Mixture
}

\author{
A.K. Singh ${ }^{a}$, R. ManohaR ${ }^{a, *}$,J.P. Shukla ${ }^{a}$ \\ AND A.M. BIRADAR ${ }^{b}$ \\ ${ }^{a}$ Department of Physics, Lucknow University, Lucknow-226 006, India \\ ${ }^{b}$ National Physical Laboratory, New Delhi, India \\ (Received April 3, 2006; revised version August 31, 2006)
}

\begin{abstract}
Measurements of ordinary refractive index, birefringence, density, and order parameter were made on a technologically important nematogen and the data obtained for its nematic and isotropic phase were reported. A modified wedge method was used for the measurement of the birefringence $(\delta n)$. The nematic-isotropic phase transition temperature matches very well as is exhibited from the value of refractive indices and densities obtained using different techniques. The optical anisotropy and density data were used to determine the order parameter and principal polarizability of the nematic mixture using the Vuks approach and their temperature dependence was discussed. The macroscopic order parameter was obtained and compared with microscopic order parameter. These two values agree very well.
\end{abstract}

PACS numbers: 61.30.--v, 42.70.Df

\section{Introduction}

The knowledge of optical anisotropy and refractive indices of liquid crystals and their temperature dependence is of large importance from the application point of view [1]. The molecular polarizabilities and their anisotropy are considered to be important molecular properties of liquid crystal substances $[2,3]$. Order parameter is uniquely important parameter of a liquid crystal, which controls nearly all its physical properties [4-7]. According to de Gennes any of the bulk tensorial properties like elastic constant, electric and magnetic susceptibilities, and refractive indices can be used to determine macroscopic order parameter [4-6]. The microscopic order parameter first introduced by Tsvetkov [8] is given by

*corresponding author; e-mail: rajlu1@rediffmail.com 
$S=\frac{1}{2}\left\langle 3 \cos ^{2} \theta-1\right\rangle$, where $\theta$ is angle between optic axis and long molecular axis of a molecule. Macroscopic order parameter $(Q)$ as determined by different properties differs among them and also differs from microscopic order parameter because the effect of the local field is different on different properties $[4-7,9]$. Several methods for the determination of the order parameter have been developed due to its great importance in understanding various physical properties of mesogens. Among those methods, optical methods are most commonly used [9-13]. Most of the investigators agree that the data obtained by the optical measurements are most accurate, precise and convenient. Thus optical methods are normally preferred to determine the order parameter. The optical anisotropy data are also important for application of any liquid crystal in display devices as it governs the thickness of liquid crystal layer [14]. Low birefringence and high order parameter liquid crystals are needed for practical application [15].

Order parameter of large number of pure nematic liquid crystals have been reported using optical anisotropy data [9-13] by large number of workers. However, there have been relatively a few studies reported on mixtures [16-18]. Therefore, we have undertaken the present study on the variation of refractive indices $\left(n_{\mathrm{e}}, n_{\mathrm{o}}\right)$, optical anisotropy $(\delta n)$, density and order parameter with temperature, for nematic mixture E-24 [19].

\section{Experimental detail}

\subsection{The material}

The sample undertaken for present investigation: nematic mixture E-24 [19] was supplied by BDH, England and used as such. The transition scheme of the sample is as follows:

crystal $\underset{-5{ }^{\circ} \mathrm{C}}{\stackrel{268 \mathrm{~K}}{\longrightarrow}}$ nematic $\underset{54^{\circ} \mathrm{C}}{\stackrel{327}{\longrightarrow}}$ isotropic.

This particular sample has great practical application due to its long temperature range of nematic phase near room temperature.

\subsection{Refractive indices measurement}

Ordinary refractive index $\left(n_{\mathrm{o}}\right)$ has been measured using Abbe refractometer having an accuracy of 0.0001 [20]. A polarizer has been introduced in Abbe refractometer to block the extraordinary ray, which improves the contrast of the boundary line at view of refractometer. Since extraordinary refractive index was larger than the refractive index of glass prism of refractometer and therefore is out of range of refractometer, which is $1.3-1.7$, some other technique has to be applied for its determination. The wedge method [7, 13] modified by Sarna et al. [21] has been used in the present work for determination of extraordinary component of refractive index $\left(n_{\mathrm{e}}\right)$. The accuracy in determination of $\delta n$ with this is $\pm 0.5 \%$. The temperature of the sample was varied by placing the wedge on a specially designed double walled brass chamber and circulating heated oil using 
Julabo F-25 refrigerated circulator. It has the facility of setting the temperature of the sensor used and so the temperature of the sample i.e. the sensor temperature has been measured directly from the display of the monitor [22]. The temperature was measured by placing a thermocouple in close vicinity of the sample with an accuracy of $\pm 0.1^{\circ} \mathrm{C}$.

\subsection{Density measurement}

The density of the nematic liquid crystal mixture E-24 at varying temperatures has been measured using a dilatometer filled with sample and a travelling microscope of least count $0.001 \mathrm{~cm}$ to read the change in the meniscus during thermal expansion [21]. The weight of the sample has been taken by an electronic balance (Sartorius BP121) having least count of $0.0001 \mathrm{~g}$. The temperature of the sample was regulated using a double walled glass jacket fitted with Julabo F-25.

\section{Theory}

As already mentioned, a homogeneously aligned nematic layer behaves like uniaxial crystal. The relationship between refractive index parallel and perpendicular to the direction of molecular axis and macroscopic order parameter $(Q)$ can be obtained by modifying the appropriate equation for uniaxial crystal [16] as

$$
\begin{aligned}
& n_{\|}=\bar{n}+\frac{2}{3} Q \Delta n, \\
& n_{\perp}=\bar{n}-\frac{1}{3} Q \Delta n,
\end{aligned}
$$

where $\bar{n}$ is the average refractive index and $\Delta n$ is the birefringence corresponding to complete alignment and for uniaxial crystal $n_{\|}=n_{\mathrm{e}}$ and $n_{\perp}=n_{\mathrm{o}}[23,24]$. From both Eqs. (1) and (2), we get

$$
Q=\frac{n_{\|}-n_{\perp}}{\Delta n}=\frac{n_{\mathrm{e}}-n_{\mathrm{o}}}{\Delta n}=\frac{\delta n}{\Delta n},
$$

where $\delta n=n_{\mathrm{e}}-n_{\mathrm{o}}$.

The value of macroscopic order parameter equal to 1 represents complete order at absolute temperature, that is at $0 \mathrm{~K} \delta n=\Delta n$. Therefore the macroscopic order parameter $(Q)$ has been obtained by extrapolating $\delta n$ for $T=0 \mathrm{~K}$. This extrapolation is done on the linear portion of the graph drawn between birefringence $(\delta n)$ against $\ln \left(1-T / T_{\mathrm{c}}\right)$ as evaluated by others $[16,18]$.

In order to find out the microscopic order parameter $(S)$, the refractive indices $n_{\mathrm{e}}$ and $n_{\mathrm{o}}$ have been analyzed following the method of Haller [25] and Horn [26]. This method uses the Vuks relation [27]:

$$
S\left(\frac{\delta \alpha}{\alpha}\right)=\frac{3\left(n_{\mathrm{e}}^{2}-n_{\mathrm{o}}^{2}\right)}{n_{\mathrm{e}}^{2}+2 n_{\mathrm{o}}^{2}-3},
$$

where $\delta \alpha$ is the anisotropy of polarizability and $\alpha$ the mean molecular polarizability. To determine $S$ we plot $\frac{3\left(n_{\mathrm{e}}^{2}-n_{\mathrm{o}}^{2}\right)}{n_{\mathrm{e}}^{2}+2 n_{\mathrm{o}}^{2}-3}$ against $\ln \left(1-T / T_{\mathrm{c}}\right)$ and extrapolate 
the linear portion of graph for $T=0 \mathrm{~K}$. The intercept at $T=0 \mathrm{~K}$, where the complete order occurs i.e. $S=1$ gives the value of $\left(\frac{\delta \alpha}{\alpha}\right)$. Inserting the value of $\left(\frac{\delta \alpha}{\alpha}\right)$ in Eq. (3) gives the value of microscopic order parameter $(S)$ for different temperatures. The principal polarizabilities have been evaluated as described by Sarna et al. [21].

We have also calculated $\alpha_{\mathrm{e}}$ and $\alpha_{\mathrm{o}}$ using well known following equations:

$$
\alpha_{\mathrm{e}, \mathrm{o}}=\frac{3}{4 \pi N} \frac{n_{\mathrm{e}, \mathrm{o}}^{2}-1}{\overline{n^{2}}+2} \text {. }
$$

\section{Result and discussion}

The temperature variation of ordinary and extraordinary refractive indices $\left(n_{\mathrm{e}}, n_{\mathrm{o}}\right)$ along with birefringence $(\delta n)$ is shown in Fig. 1 . In isotropic phase the birefringence $(\delta n)$ is zero and refractive index decreases with increase in temperature like any other normal organic liquid [28]. The ordinary refractive index $\left(n_{\mathrm{o}}\right)$ increases slightly while the extraordinary refractive index $\left(n_{\mathrm{e}}\right)$ decreases sharply with increase in temperature. At nematic-isotropic phase transition point, i.e. at $327 \mathrm{~K}$, the refractive indices values change abruptly. The birefringence values also show sharp decrease with increase in temperature. Its value 0.24 at $298 \mathrm{~K}$ reduces to almost 0.1 at $324 \mathrm{~K}$. Similar type of behavior has also been reported for nematic mixture by several other workers [16-18].

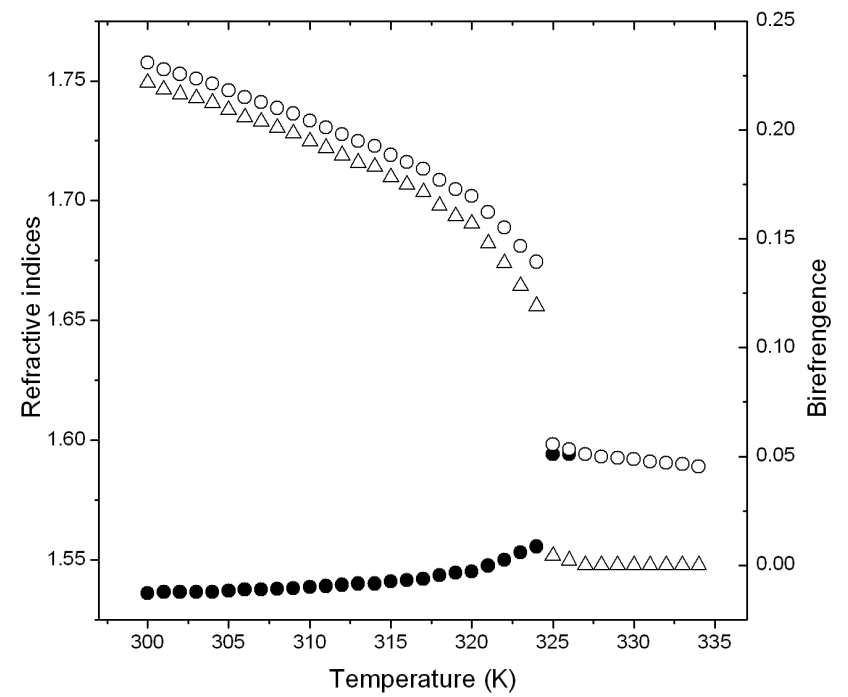

Fig. 1. Variation of refractive indices $n_{\mathrm{e}}(\circ), n_{\mathrm{o}}(\bullet)$ and birefringence $\delta n(\Delta)$ with the temperature.

However, phase transition temperature of the mixture E-24 measured is not as sharp as in a pure (single component) nematic sample. The breadth of the 
transition from nematic to isotropic phase was found as $6 \mathrm{~K}$. Demus et al. [29] and Singh et al. [16] have also observed similar behaviour and concluded that the breadth of the transition depends upon purity of the substance and it increases with lower purity. Cheng and Meyer [30] have reported pre-transition temperature of $11 \mathrm{~K}$ in a mesogen. In the case of our sample, which is a nematic mixture, the different components behave, as impurity with respect to each other and that might be the reason for biphasic region.

The density of the sample (Fig. 2) decreases almost linearly with increasing temperature like in pure nematic samples $[31,21]$. The phase transition is indicated by a simple discontinuity of $6 \mathrm{~K}$ transition width suggesting first order phase transition [32]. The slightly broad breadth of transition is due to the different components of the mixture as mentioned above. However, the phase transition temperature for the nematogen, as obtained by different techniques, i.e. refractive indices measurement technique and density measurement technique, are in good agreement.

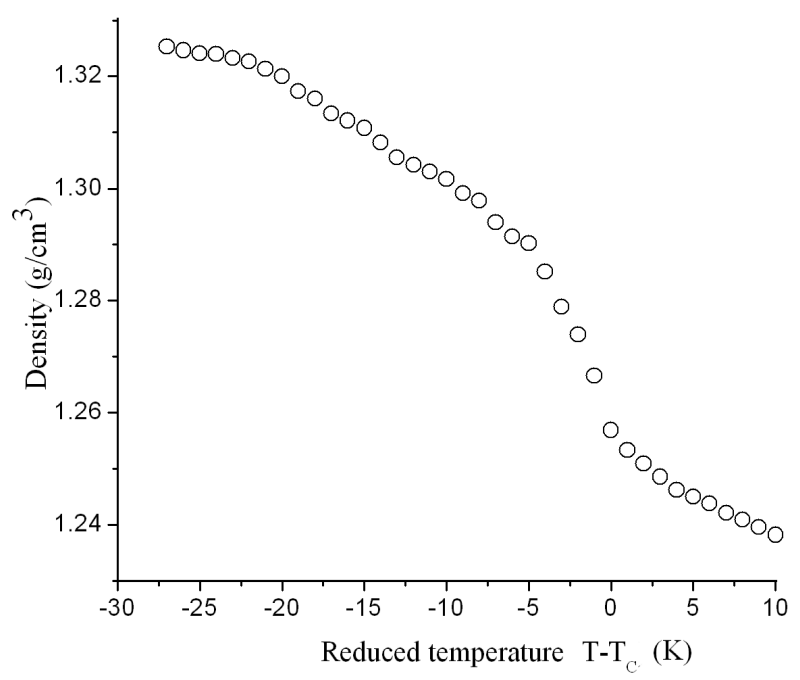

Fig. 2. Temperature variation of density.

The variation of the ratio of principal polarizability with temperature as shown in Fig. 3 decreases with increase in temperature and after the phase transition has reached its value becomes 1 , which indicates that the sample has become isotropic. The variation of $\left(\alpha_{\mathrm{e}} / \alpha_{\mathrm{o}}\right)$ is essentially due to the variation in order parameter. It suggests that orientation of long molecular axis with director changes and the value of $\theta$ increases with increase in temperature and the sample becomes more disordered.

As can be seen from Fig. 4, the microscopic order parameter $(S)$ as calculated from refractive indices data using the Vuks approach (relation) matches very well 


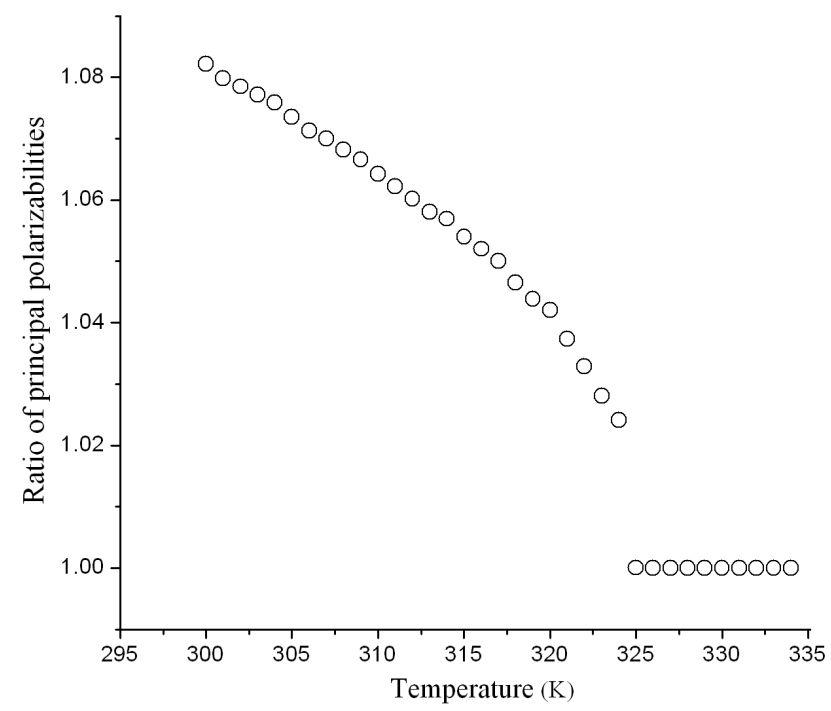

Fig. 3. Temperature variation of ratio of principal polarizabilities.

with macroscopic order parameter $(Q)$ for nematic mixture E-24. Kuczyński [33] has suggested that one can expect that within a given local field they may be found to be nearly the same as is seen in the present study.

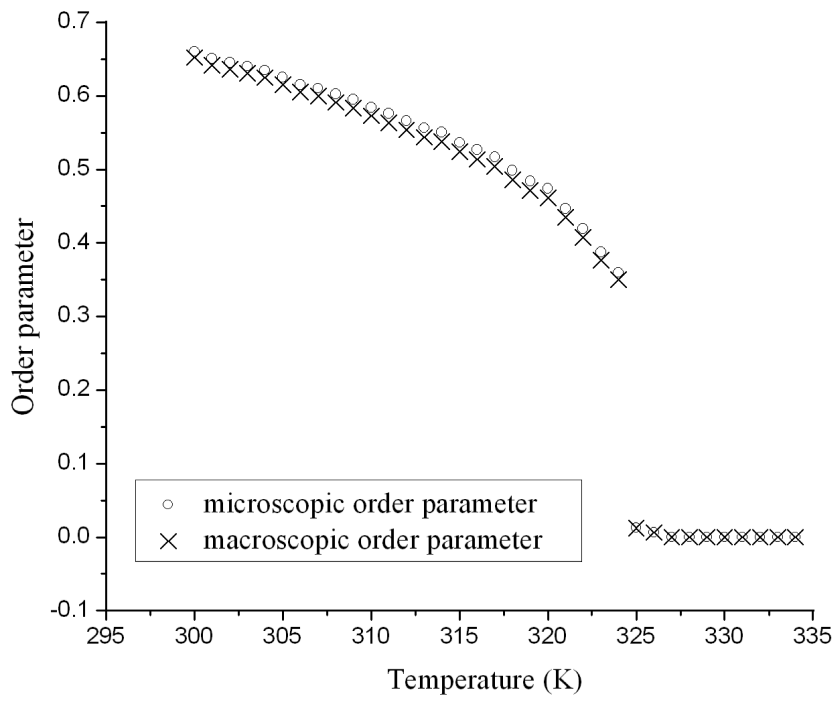

Fig. 4. Temperature variation of microscopic $(S)$ and macroscopic $(Q)$ order parameter.

The value of both order parameters decreases as temperature increases and shows a sharp change at nematic-isotropic transition temperature. It decreases gradually from 0.67 at $300 \mathrm{~K}$ to 0.35 at $324 \mathrm{~K}$ and then becomes 0 . The behaviour 
TABLE

Refractive indices, density, microscopic and macroscopic order parameter values of the nematogen E-24 for different temperatures.

\begin{tabular}{|c|c|c|c|c|c|}
\hline $\begin{array}{c}\text { Temperature } \\
{[\mathrm{K}]}\end{array}$ & $\begin{array}{l}\text { Ordinary } \\
\text { refractive } \\
\text { index }\left(n_{\mathrm{o}}\right)\end{array}$ & $\begin{array}{c}\text { Extraordinary } \\
\text { refractive } \\
\text { index }\left(n_{\mathrm{e}}\right)\end{array}$ & $\begin{array}{l}\text { Density } \\
{\left[\mathrm{g} / \mathrm{cm}^{3}\right]}\end{array}$ & $\begin{array}{c}\text { Macroscopic } \\
\text { order } \\
\text { parameter }(Q)\end{array}$ & $\begin{array}{c}\text { Microscopic } \\
\text { order } \\
\text { parameter }(S)\end{array}$ \\
\hline 300 & 1.5360 & 1.7576 & 1.3253 & 0.6519 & 0.6602 \\
\hline 301 & 1.5365 & 1.7548 & 1.3246 & 0.6421 & 0.6507 \\
\hline 302 & 1.5365 & 1.7528 & 1.3241 & 0.6363 & 0.6453 \\
\hline 303 & 1.5365 & 1.7509 & 1.3239 & 0.6305 & 0.6400 \\
\hline 304 & 1.5365 & 1.7489 & 1.3233 & 0.6247 & 0.6346 \\
\hline 305 & 1.5370 & 1.7460 & 1.3226 & 0.6148 & 0.6251 \\
\hline 306 & 1.5375 & 1.7432 & 1.3213 & 0.6050 & 0.6155 \\
\hline 307 & 1.5375 & 1.7412 & 1.3200 & 0.5991 & 0.6101 \\
\hline 308 & 1.5378 & 1.7387 & 1.3173 & 0.5909 & 0.6022 \\
\hline 309 & 1.5380 & 1.7364 & 1.3160 & 0.5834 & 0.5950 \\
\hline 310 & 1.5386 & 1.7333 & 1.3134 & 0.5727 & 0.5845 \\
\hline 311 & 1.5390 & 1.7306 & 1.3121 & 0.5635 & 0.5756 \\
\hline 312 & 1.5395 & 1.7277 & 1.3108 & 0.5536 & 0.5659 \\
\hline 313 & 1.5400 & 1.7249 & 1.3081 & 0.5437 & 0.5562 \\
\hline 314 & 1.5400 & 1.7229 & 1.3056 & 0.5378 & 0.5506 \\
\hline 315 & 1.5410 & 1.7191 & 1.3043 & 0.5238 & 0.5365 \\
\hline 316 & 1.5415 & 1.7162 & 1.3030 & 0.5137 & 0.5266 \\
\hline 317 & 1.5420 & 1.7133 & 1.3017 & 0.5038 & 0.5168 \\
\hline 318 & 1.5435 & 1.7086 & 1.2991 & 0.4855 & 0.4982 \\
\hline 319 & 1.5445 & 1.7047 & 1.2978 & 0.4713 & 0.4840 \\
\hline 320 & 1.5450 & 1.7018 & 1.2940 & 0.4613 & 0.4741 \\
\hline 321 & 1.5475 & 1.6953 & 1.2915 & 0.4346 & 0.4467 \\
\hline 322 & 1.5500 & 1.6887 & 1.2902 & 0.4078 & 0.4193 \\
\hline 323 & 1.5530 & 1.6811 & 1.2852 & 0.3767 & 0.3874 \\
\hline 324 & 1.5555 & 1.6744 & 1.2789 & 0.3498 & 0.3597 \\
\hline 325 & 1.5940 & 1.5982 & 1.2740 & 0.0124 & 0.0127 \\
\hline 326 & 1.5940 & 1.5961 & 1.2666 & 0.0062 & 0.0063 \\
\hline 327 & 1.5940 & 1.5940 & 1.2569 & 0.0000 & 0.0000 \\
\hline 328 & 1.5930 & 1.5930 & 1.2533 & 0.0000 & 0.0000 \\
\hline 329 & 1.5925 & 1.5925 & 1.2509 & 0.0000 & 0.0000 \\
\hline 330 & 1.5920 & 1.5920 & 1.2486 & 0.0000 & 0.0000 \\
\hline 331 & 1.5910 & 1.5910 & 1.2462 & 0.0000 & 0.0000 \\
\hline 332 & 1.5905 & 1.5905 & 1.2450 & 0.0000 & 0.0000 \\
\hline 333 & 1.5900 & 1.5900 & 1.2439 & 0.0000 & 0.0000 \\
\hline 334 & 1.5890 & 1.5890 & 1.2421 & 0.0000 & 0.0000 \\
\hline
\end{tabular}


of order parameter curve is almost the same as for the normal nematic sample $[17,18,31,34,33]$. The refractive indices, density, microscopic and macroscopic order parameter values have been given in Table at different temperatures.

The values of birefringence, refractive indices, order parameter etc. and their dependence is found to be similar to those obtained by other workers for nematic mixtures like ZLI-1565, 1333, RO-TN 605, E-43 [35], ZLI-2788-000, and ZLI-2222-000 [18].

\section{Conclusion}

It may be concluded that the phase transition temperature as obtained by two different techniques are in close agreement for the nematic mixture E-24. However, a broader breadth of range $6 \mathrm{~K}$ of phase transition for density is due to the biphasic region, which shows existence of different components in the sample. The macroscopic and microscopic order parameters are almost the same in both the nematic and isotropic phase as expected because they have been determined by using a single local field model.

\section{References}

[1] Liquid Crystals: Applications and Uses, Vol. 1, Ed. B. Bahadur, World Sci., New Jersey, USA 1990.

[2] W.H. de Jeu, Mol. Cryst. Liq. Cryst. 63, 83 (1980).

[3] N.V.S. Rao, V.G.K.M. Pisipati, P.V. Datta Prasad, P.R. Alapati, Mol. Cryst. Liq. Cryst. 132, 1 (1986).

[4] P.G. de Gennes, The Physics of Liquid Crystal, Clarendon Press, Oxford 1974, p. 3 .

[5] S. Chandrasekhar, Liquid Crystals, Cambridge University Press, Cambridge 1977; Rep. Prog. Phys. 39, 613 (1976).

[6] M.J. Stephen, J.P. Straley, Rev. Mod. Phys. 46, 193 (1974).

[7] E.G. Hanson, Y.R. Shen, Mol. Cryst. Liq. Cryst. 36, 132 (1976).

[8] V. Tsvetkov, Acta Physicochim. (USSR) 16, 132 (1942).

[9] E.G. Hanson, Y.R. Shen, Mol. Cryst. Liq. Cryst. 36, 193 (1979).

[10] I. Haller, H.A. Huggins, H.R. Lilienthal, T.R. McGuire, J. Phys. Chem. 77, 950 (1973).

[11] I.H. Ibrahim, W. Haase, Z. Naturforsch. 319, 1644 (1979).

[12] W.H. de Jeu, P. Bordewijk, J. Chem. Phys. 68, 109 (1978).

[13] I. Haller, H.A. Huggins, M.H. Freiser, Mol. Cryst. Liq. Cryst. 16, 53 (1972).

[14] F.V. Allan, Information Displays 10, 14 (1983).

[15] H.S. Cole, S. Aftergut, J. Chem. Phys. 68, 896 (1978).

[16] R. Singh, V.K. Agrawal, P.P. Anand, V.P. Arora, Natl. Acad. Sci. Lett. (India) 13, 129 (1990).

[17] M. Mitra, S. Paul, R. Paul, Pramana - J. Phys. 29, 409 (1987). 
[18] V.P. Arora, S. Prakash, V.K. Agrawal, B. Bahadur, Ind. J. Pure Appl. Phys. 30, 406 (1992).

[19] BDH Catalog, BDH Chemical Ltd., Poole BH 12 4NN, England 1978, p. 19.

[20] Li Jun, S. Gauzia, S.T. Wu, Optics Express 12, 9 (2004).

[21] R.K. Sarna, B. Bahadur, V.G. Bhide, Mol. Cryst. Liq. Cryst. 51, 117 (1979).

[22] Operating Manual, JULABO, Labortechnik Gmbh, p. 17.

[23] I. Muševič, I. Drevenšek, R. Blinc, S. Kumar, J.W. Doane, Mol. Cryst. Liq. Cryst. 172, 217 (1989).

[24] W.H. de Jeu, Physical Properties of Liquid Crystalline Materials, Gordon and Breach, New York 1980.

[25] I. Haller, Prog. Solid State Chem. (GB) 10, 103 (1975).

[26] R.G. Horn, J. Phys. (France) 39, 105 (1978).

[27] M.F. Vuks, Opt. Spectrosc. (USA) 20, 361 (1966).

[28] Handbook of Physics and Chemistry $(C R C)$, 57th ed., CRC Press, Cleveland, Ohio 1976, p. 77; C.P. Smyth, Dielectric Behaviour and Structure, McGraw-Hill, New York 1975.

[29] D. Demus, H.G. Hahn, F. Kuschel, Mol. Cryst. Liq. Cryst. 44, 61 (1978).

[30] J. Cheng, R.B. Meyer, Phys. Rev. A 9, 2744 (1974).

[31] K. Bhowmick, A. Mukhopadhyay, C.D. Mukherjee, Mol. Cryst. Liq. Cryst. 366, 229 (2001).

[32] N.H. Harston, A. Stuart, Crystals and Polarizing Microscope, Edward Arnold, London 1970.

[33] W. Kuczyński, B. Żywucki, J. Malecki, Mol. Cryst. Liq. Cryst. 381, 1 (2002).

[34] S. Sen, P. Brahma, K. Roy Subik, D.K. Mukherjee, S.B. Roy, Mol. Cryst. Liq. Cryst. 100, 327 (1983).

[35] A.K. Garg, V.K. Agarwal, B. Bahadur, Mol. Cryst. Liq. Cryst. 130, 11 (1985). 